J. Austral. Math. Soc. (Series A) 42 (1987), 57-68

\title{
ON THE CONVEXITY AND SYMMETRY OF SOLUTIONS TO AN ELLIPTIC FREE BOUNDARY PROBLEM
}

\author{
BERNHARD KAWOHL \\ Dedicated to Professor W. Wendland on the occasion of his 50 th birthday
}

(Received 15 October 1984; revised 6 May 1985)

Communicated by R. O. Vyborny

\begin{abstract}
We study the existence, uniqueness and regularity of solutions to an exterior elliptic free boundary problem. The solutions model stationary solutions to nonlinear diffusion reaction problems, that is, they have compact support and satisfy both homogeneous Dirichlet and Neumann-type boundary conditions on the free boundary $\partial\{u>0\}$. Then we prove convexity and symmetry properties of the free boundary and of the level sets $\{u>c\}$ of the solutions. We also establish symmetry properties for the corresponding interior free boundary problem.
\end{abstract}

1980 Mathematics subject classification (Amer. Math. Soc.): 35 J 65, 35 J 20, 35 B 50, 26 D 10.

\section{Introduction}

Consider the exterior boundary value problem

$$
\begin{gathered}
\Delta u=\lambda f(u) \quad \text { in } \mathbb{R}^{n} \backslash \bar{\Omega}, \lambda>0, \\
u \equiv 1 \quad \text { on } \bar{\Omega}, \quad u(x) \rightarrow 0 \quad \text { as }|x| \rightarrow \infty,
\end{gathered}
$$

with $\Omega$ a bounded domain. Under suitable assumptions on $f$, one can show that this problem has solutions with compact support. We shall prove that the symmetry and convexity properties of $\Omega$ are "inherited" by the support of any solutions to (1.1), (1.2). Our study was motivated by a recent paper of Friedman and Phillips (1984) on the corresponding interior problem. The relevance of such

(C) 1987 Australian Mathematical Society $0263-6115 / 87 \$ A 2.00+0.00$ 
free boundary problems is described for example in Bandle, Sperb and Stakgold (1984), Diaz and Herrero (1981), Friedman and Phillips (1984) or Frank and Wendt (1984).

This paper is organized as follows. In Section 1 we discuss existence and uniqueness of solutions by variational methods and introduce some notation. In Section 2 we modify the so called Gabriel-Lewis method to prove convexity of all level sets $\Omega_{c}:=\left\{x \in \mathbf{R}^{n} \mid u(x)>c\right\}$ of solutions to (1.1), (1.2). In Section 3 we discuss the symmetry properties of variational solutions. Finally in Section 4 we comment on the corresponding interior problem.

\section{Existence and regularity}

Let $\Omega \subset \mathbb{R}^{n}$ be a bounded domain with boundary $\partial \Omega$ of class $C^{2, \alpha}$. We want to state existence, uniqueness and regularity results for the exterior problem (1.1), (1.2). It will be convenient to refer to one or several of the following assumptions about $f$.

$$
\begin{aligned}
& f(t)=0 \text { for } t \leqslant 0, f(t)>0 \text { for } t>0, f \sim t^{\alpha} \\
& \text { as } t \downarrow 0(0<\alpha<1) \text { and } f \in C^{1}((0,1]) . \\
& f \text { is monotone, } f(t)=0 \text { for } t \leqslant 0, f(t)>0 \\
& \text { for } 0<t \leqslant 1 \text {. } \\
& \quad f \in C^{1, \beta}((0,1)) \text { for some } \beta \in(0,1) . \\
& \int_{0}^{1} F(t)^{-1 / 2} d t<\infty, \text { where } F(t)=\int_{0}^{t} f(\tau) d \tau .
\end{aligned}
$$

Notice that a mapping $f$ satisfying (1.4)-(1.6) might have a jump discontinuity at zero and that (1.3) implies (1.6). A standard example of a function $f$ satisfying (1.3) or (1.4)-(1.6) is $f(t)=\left(t^{+}\right)^{p}, 0<p<1$. The following results appear to be more or less well known.

Proposition 1.1. If $f$ satisfies (1.4), (1.5) and (1.6), then there exists a unique solution $u \in C^{3, \beta}(D) \cap C^{1, \lambda}\left(\mathbb{R}^{n} \backslash \Omega\right)$ to the exterior problem (1.1), (1.2). Here $D:=\left\{x \in \mathbb{R}^{n} \mid 0<u(x)<1\right\}$, and $\lambda \in(0,1)$.

Proposition 1.2. If $f$ satisfies (1.3), then there exists a classical solution $u \in C^{2, \alpha}\left(\mathbb{R}^{n} \backslash \bar{\Omega}\right)$, with compact support, to the exterior problem (1.1), (1.2).

Let us give the proof of Proposition 1.1 in some detail and only sketch the proof of Proposition 1.2. 
Proof of Proposition 1.1. Since by the maximum principle $u$ will only assume values between 0 and 1 , we may alter the mapping $f$ outside the unit interval and introduce a maximal monotone mapping $\beta$ on $\mathbb{R}$ with the properties $\beta(u)=\{f(u)\}$ for $u$ in $(0,1],-\beta(-u)=\beta(u)$ on $\mathbb{R}$ and $R(\beta)=\mathbb{R}$. In Diaz and Herrero (1981) one can find that the modified problem

$$
\begin{gathered}
\Delta u \in \beta(u) \quad \text { in } \mathbf{R}^{n} \backslash \bar{\Omega}, \\
u \equiv 1 \quad \text { on } \bar{\Omega}, \quad u(x) \rightarrow 0 \quad \text { as }|x| \rightarrow \infty,
\end{gathered}
$$

has a unique weak solution $u \in W^{1, p}\left(\mathbf{R}^{n} \backslash \bar{\Omega}\right)$ with compact support, $1<p<\infty$. Condition (1.6) is needed to prove the compactness of supp $u$. In the domain $D$ the solution $u$ of problem (1.7), (1.2) also satisfies equation (1.1) and is therefore a solution to (1.1), (1.2). The uniqueness follows from standard monotonicity arguments because $u$ is a solution to the variational problem

$$
\operatorname{Min}_{\substack{v \in H^{1}(B) \\ v=1 \text { on } \partial \Omega \\ v=0 \text { on } \partial B}} J_{\text {ext }}(v):=\int_{B}\left\{\frac{1}{2}|\nabla v(x)|^{2}+F(v(x))\right\} d x,
$$

where $B$ is a sufficiently large ball containing $D$, and because $J_{\text {ext }}$ is coercive and convex under assumption (1.4). The $C^{3, \beta}$ regularity of $u$ in $D \backslash \bar{\Omega}$ is a consequence of (1.5). Since $f$ is bounded on $[0,1]$, we may interpret (1.1) as a linear equation with $L^{\infty}$ right-hand side and so obtain $W^{2, \infty}$-regularity of $u$ in $\mathbb{R}^{n} \backslash \bar{\Omega}$.

The last assertion of Proposition 1.1 follows now from Sobolev's embedding theorem.

Before we proceed with the proof of Proposition 1.2, let us define variational solutions of our problem and make some heuristic remarks.

We call $u \in H^{1}\left(R^{n}\right)$ a variational solution of the exterior problem (1.1), (1.2) if it solves (1.8). Clearly any variational solution of the exterior problem satisfies (1.1) in a weak sense and, if (1.3) is assumed, constitutes a classical solution. So once we prove the existence of a variational solution $u$, to the exterior problem, with compact support, the proof of Proposition 1.2 is complete. We are faced with the difficulty that the function $F$ in (1.8) does not have to be convex. Suppose we replace $F$ by $\tilde{F}$, where $\tilde{F}$ is convex and $\tilde{F} \leqslant F$, and suppose that we can show that there exists a (necessarily unique) variational solution $\tilde{u}$ of this modified problem. Then we expect $\tilde{u}$ to have larger support than $u$ for the following reason. The term $\int_{\Omega} \tilde{F}(v(x)) d x$ can be interpreted as a penalty term for the support of $v$, and there is a smaller penalty on the support of $v$ if we replace $F$ by $\tilde{F}$. This idea led us to the following

Proof of Proposition 1.2. We replace $f(t)$ by $\tilde{f}(t):=\min _{s \geqslant t} f(s)$. Obviously, $\tilde{f}(t) \leqslant f(t)$, and $\tilde{f}(t)$ is a monotone function of class $C^{\alpha}(\mathbb{R})$. Consequently, we may proceed as in the proof of Proposition 1.1. We have to observe 
that $\tilde{f}$ satisfies (1.6), because $f$ satisfies (1.3), and we obtain a solution $\tilde{u} \in$ $C^{2, \alpha}\left(\mathbf{R}^{n} \backslash \bar{\Omega}\right)$, with compact support, of the modified problem $\Delta \tilde{u}=\tilde{f}(\tilde{u})$ in $\mathbf{R}^{n} \backslash \bar{\Omega}, \tilde{u} \equiv 1$ on $\bar{\Omega}$. From a comparison argument, we may now conclude that $u \leqslant \tilde{u}$ for any classical solution of (1.1), (1.2). If a classical solution $\tilde{u}$ of (1.1), (1.2) exists, it will have zero boundary values on the boundary of supp $\tilde{u}$. Hence we have reduced problem (1.1), (1.2) to a semilinear Dirichlet problem on a bounded domain which is solvable by standard arguments.

Let us summarize what we know about the exterior problem. There exist solutions $u$ with compact support. The boundary of the support is called the free boundary, and we shall denote it by $\Gamma$. Since $u$ is of class $C^{1}$ across the free boundary, $u$ formally satisfies:

$$
u=0 \text { and } \frac{\partial u}{\partial n}=0 \text { on } \Gamma .
$$

If $\Gamma$ is singular, then the normal derivative of $u$ might not be well defined. For Hölder continuous functions $f$, the $C^{1}$-regularity of $\Gamma$ was established by Friedman and Phillips (1984), but we allow $f$ to be discontinuous. Aside from their results, little seems to be known about the shape of the free boundary. In Kawohl (1983b) the following result was derived by a rearrangement method.

Proposition 1.3. Let $\Omega$ and f satisfy the assumptions of Proposition 1.1 or 1.2 .

(a) If $\Omega$ is starshaped with respect to a point $x^{0} \in \Omega$, then so also is the support of u.

(b) If $\Omega$ is starshaped with respect to each point $x$ in an $\varepsilon$-neighborhood of zero, then the free boundary is Lipschitz continuous.

Notice that the assumptions of (b) are satisfied without loss of generality for convex domains with nonempty interior, since problem (1.1), (1.2) is translation invariant. For Lipschitz continuous free boundaries, the normal derivative is defined almost everywhere, and hence boundary condition (1.9) makes sense and has to be understood a.e. on $\Gamma$.

\section{Convexity of the free boundary for the exterior problem}

THEOREM 2.1. If $f$ satisfies (1.4), (1.5) and (1.6), and if $\Omega \subset \mathbb{R}^{n}$ is a convex, bounded domain with nonempty interior, then all the level sets $\Omega_{c}:=\left\{x \in \mathbb{R}^{n} \mid u(x)\right.$ $>0$ ) of the solution $u$ to problem (1.1), (1.2), and in particular the support of $u$, are convex. 
Proof. This proof is an extension of the so-called Gabriel-Lewis method. A continuous function $u$ on a convex domain $C$ is known to have convex level sets $\{u \geqslant c\}$ if and only if an auxiliary function $Q$ possesses the right sign:

$$
Q\left(x_{1}, x_{2}\right):=u\left(\frac{1}{2}\left(x_{1}+x_{2}\right)\right)-\min \left\{u\left(x_{1}\right), u\left(x_{2}\right)\right\} \geqslant 0 \text { in } C \times C .
$$

In this case we call $u$ quasiconcave. The strategy of the proof consists in verifying (2.1). To this end let $C:=$ the open convex hull of $D$. Once we manage to show that $C=\bar{\Omega} \cup D$, we may then apply arguments from Kawohl (1983a) to conclude that other level sets of $u$ are convex. If (2.1) is violated, then there exists a pair of points $\left(y_{1}, y_{2}\right) \in \bar{C} \times \bar{C}$ such that

$$
Q \text { attains a global negative minimum at }\left(y_{1}, y_{2}\right) \text {, and }
$$

$$
u\left(\frac{1}{2}\left(y_{1}+y_{2}\right)\right)<\min \left\{u\left(y_{1}\right), u\left(y_{2}\right)\right\}
$$

and since $u \geqslant 0$ in $C$ and $u=0$ on $\partial C$, we already know

$$
y_{1} \text { and } y_{2}\left(\text { and } \frac{y_{1}+y_{2}}{2}\right) \text { are in } C \text {. }
$$

The next steps in the proof are aimed at showing that $y_{1}, y_{2}$ and $\left(y_{1}+y_{2}\right) / 2$ are all in $D$ (Lemmas 2.4(b) and 2.6(b)). In Kawohl (1983a) one can find a proof of the following result.

LEMMA 2.3. If $\operatorname{supp} u$ and $\Omega$ are starshaped with respect to $x^{0} \in \Omega$, then $\left(x-x^{0}\right) \nabla u(x)<0$ in $D$.

Using Proposition 1.3 and the convexity of $\Omega$, we obtain

$$
\left(x-x^{0}\right) \nabla u(x)<0 \text { in } D \text { for any } x^{0} \in \Omega,
$$

and, in particular,

$$
|\nabla u(x)|>0 \text { in } D
$$

LEMMA 2.4. (a) $u\left(y_{1}\right)=u\left(y_{2}\right)>0$.

(b) $y_{1} \in D, y_{2} \in D$ and $\left(y_{1}+y_{2}\right) / 2 \in C \backslash \bar{\Omega}$.

Proof. (a) Suppose that $u\left(y_{1}\right)<u\left(y_{2}\right)$. Then in a neighborhood of $\left(y_{1}, y_{2}\right)$ the quasiconcavity function $Q$ has the $C^{2}$-representation

$$
Q\left(x_{1}, x_{2}\right)=u\left(\frac{1}{2}\left(x_{1}+x_{2}\right)\right)-u\left(x_{1}\right),
$$

and the gradients of $C$ with respect to $x_{1}$ and $x_{2}$ have to vanish at $\left(y_{1}, y_{2}\right)$, i.e.,

$$
\frac{1}{2} \nabla u\left(\frac{1}{2}\left(y_{1}+y_{2}\right)\right)=\nabla u\left(y_{1}\right)=0 \text {. }
$$


But this implies that $u\left(y_{1}\right)=0$ because of (2.5), and hence $Q\left(y_{1}, y_{2}\right) \geqslant 0$ in contrast to (2.2). Therefore $u\left(y_{1}\right)$ has to be equal to $u\left(y_{2}\right)$. If they are both zero, then again $Q\left(y_{1}, y_{2}\right) \geqslant 0$, which contradicts (2.2).

(b) We know from Lemma 2.4(a) that $y_{1} \in D \cup \bar{\Omega}$ and that $y_{2} \in D \cup \bar{\Omega}$. We have therefore to exclude the possibilities (i) $y_{1}$ and $y_{2} \in \bar{\Omega}$, and (ii) $y_{1} \in \bar{\Omega}$ and $y_{2} \in D$. In case (i) we have $\left(y_{1}+y_{2}\right) / 2 \in \bar{\Omega}$ by the convexity of $\bar{\Omega}$, and we have $Q\left(y_{1}, y_{2}\right) \geqslant 0$. In case (ii) we apply Lemma 2.3 with $x^{0}=y_{1}$ and $x=y_{2}$ and even get that $Q\left(y_{1}, y_{2}\right)>0$. Finally, $\left(y_{1}+y_{2}\right) / 2 \in \bar{\Omega}$ implies $Q\left(y_{1}, y_{2}\right) \geqslant 0$, since $u \leqslant 1$ on $\mathbb{R}^{n}$.

In order to prove that $\left(y_{1}+y_{2}\right) / 2 \in D$, we have to compare the gradients of $u$ at $y_{1}, y_{2}$ and $\left(y_{1}+y_{2}\right) / 2$. Recall that, because of Proposition 1.2 and Lemma 2.4(b), the function $u$ is differentiable at those three points.

Lemma 2.5. $\nabla u\left(y_{1}\right)$ is parallel to and has the same direction as $\nabla u\left(y_{2}\right)$.

Proof. If this were not the case, then there would exist a unit vector $\boldsymbol{\xi} \in \mathbf{R}^{n}$ such that $u_{\xi}\left(y_{1}\right)<0$ and $u_{\xi}\left(y_{2}\right)>0$. This would contradict the fact that $Q$ attains its minimum at $\left(y_{1}, y_{2}\right)$, since one could diminish $Q$ by moving $y_{1}$ in direction $-\xi$ and $y_{2}$ in direction $\xi$.

LEMMA 2.6. (a) $\nabla u\left(\left(y_{1}+y_{2}\right) / 2\right)$ does not vanish; moreover, it is parallel to and has the same direction as $\nabla u\left(y_{1}\right)\left(\right.$ or $\left.\nabla u\left(y_{2}\right)\right)$.

(b) $\left(y_{1}+y_{2}\right) / 2 \in D$.

Proof. If $h$ is a unit vector in $\mathbf{R}^{n}$ and $\boldsymbol{t}$ a small positive number, then $Q\left(y_{1}+t h, y_{2}+t h\right)-Q\left(y_{1}, y_{2}\right) \geqslant 0$. If $\nabla u\left(\left(y_{1}+y_{2}\right) / 2\right)$ would now vanish, then one could diminish $Q$ by choosing $h=\nabla u\left(y_{1}\right)$. The remainder of (a) is now obtained as in the proof of Lemma 2.5. Assertion (b) follows from (a) and from Lemma 2.4(a).

Let us summarize: up to this point we have ruled out many possible locations of the extremal triple $y_{1}, y_{2}$ and $\left(y_{1}+y_{2}\right) / 2$. The remaining alternative is that $y_{1}, y_{2}$ and $\left(y_{1}+y_{2}\right) / 2$ are all in $D$. This may be excluded because of the special structure of equation (1.1). Notice that $u$ is three times differentiable in a neighborhood of $y_{1}, y_{2}$ and $\left(y_{1}+y_{2}\right) / 2$, so that we may in fact use equation (1.1). This was sketched in Kawohl (1983a), but for the reader's convenience we shall present the details here. In the first part of the following lemma we upgrade the qualitative statements of Lemmas 2.5 and 2.6 to quantitative ones. We introduce the notation

$$
a:=\left|\nabla u\left(\frac{y_{1}+y_{2}}{2}\right)\right|, \quad b:=\left|\nabla u\left(y_{1}\right)\right| \text { and } c:=\left|\nabla u\left(y_{2}\right)\right| .
$$




\section{LEMMA 2.7.}

(a)

$$
\begin{aligned}
\frac{1}{a} & =\frac{1}{2}\left(\frac{1}{b}+\frac{1}{c}\right) . \\
\frac{1}{a^{3}} \Delta u\left(\frac{y_{1}+y_{2}}{2}\right) & \geqslant \frac{1}{2 b^{3}} \Delta u\left(y_{1}\right)+\frac{1}{2 c^{3}} \Delta u\left(y_{2}\right) .
\end{aligned}
$$

Proof. Let us define $n:=\nabla u\left(y_{1}\right) / b$ and let us fix a unit vector $h \in \mathbb{R}^{n}$ with the property

$$
h \cdot n \neq 0 \text {. }
$$

We want to move from $y_{1}$ to $y_{1}+s h / b$, and from $y_{2}$ to $y_{2}+r(s) h / c$, in such a way that the side constraint $u\left(x_{1}\right)=u\left(x_{2}\right)$ remains satisfied. Notice that because of Lemma 2.4 the points $y_{1}, y_{2}$ provide a solution to the constrained minimization problem

$$
\text { minimize } u\left(\frac{x_{1}+x_{2}}{2}\right)-u\left(x_{1}\right) \text { subject to } u\left(x_{1}\right)=u\left(x_{2}\right) \text {. }
$$

Since $|\nabla u| \neq 0$ in $D$ (see (2.5)), and since $u \in C^{3}(D)$, there exists a $C^{2}$-function $r(s)$ such that for every small real number $s$, the relation

$$
u\left(y_{1}+\frac{s}{b} h\right)=u\left(y_{2}+\frac{r(s)}{c} h\right)
$$

holds. This follows from the implicit function theorem. We differentiate (2.8) with respect to $s$ to obtain

$$
\frac{1}{b} u_{h}\left(y_{1}+\frac{s}{b} h\right)=\frac{r^{\prime}(s)}{c} u_{h}\left(y_{2}+\frac{r(s)}{c} h\right),
$$

and so for $s=0$ we have

$$
r^{\prime}(0)=1 \text {. }
$$

Another differentiation of (2.9) at $s=0$ gives

$$
\frac{1}{b^{2}} u_{h h}\left(y_{1}\right)=\frac{1}{c^{2}} u_{h h}\left(y_{2}\right)+\frac{r^{\prime \prime}(0)}{c} u_{h}\left(y_{2}\right) .
$$

Now let us consider the auxiliary function

$$
\tilde{Q}(s, h)=Q\left(y_{1}+s h / b, y_{2}+r(s) h / c\right) .
$$

For every $h \in \mathbb{R}^{n}$, the function $\tilde{Q}$ attains a negative minimum at $s=0$. Therefore $\partial \tilde{Q} / \partial s$ has to vanish, and we have $\partial^{2} \tilde{Q} / \partial^{2} s \geqslant 0$. Let us calculate these two 
quantities and observe (2.7). First, we have

$$
\begin{aligned}
\frac{\partial \tilde{Q}}{\partial s}(s, h)= & \frac{1}{2}\left(\frac{1}{b}+\frac{r^{\prime}(s)}{c}\right) u_{h}\left(\frac{y_{1}+y_{2}}{2}+\frac{1}{2}\left(\frac{s}{b}+\frac{r(s)}{c}\right) h\right) \\
& -\frac{1}{b} u_{h}\left(y_{1}+\frac{s}{b} h\right) .
\end{aligned}
$$

For $s=0$ this gives the first assertion of Lemma 2.7. Second, we have

$$
\begin{aligned}
\frac{\partial^{2} \tilde{Q}}{\partial s^{2}}(0, h)= & {\left[\frac{1}{2}\left(\frac{1}{b}+\frac{1}{c}\right)\right]^{2} u_{h h}\left(\frac{y_{1}+y_{2}}{2}\right) } \\
& +\frac{1}{2} \frac{r^{\prime \prime}(0)}{c} u_{h}\left(\frac{y_{1}+y_{2}}{2}\right)-\frac{1}{b^{2}} u_{h h}\left(y_{1}\right) \geqslant 0 .
\end{aligned}
$$

A combination of (2.13), (2.11) and Lemma 2.7(a) now yields

$$
\frac{1}{a^{3}} u_{h h}\left(\frac{y_{1}+y_{2}}{2}\right) \geqslant \frac{1}{2 b^{3}} u_{h h}\left(y_{1}\right)+\frac{1}{2 c^{3}} u_{h h}\left(y_{2}\right) \text {, }
$$

and summation over $n$ orthogonal unit vectors $h$ will complete the proof of Lemma 2.7.

Now we are in a position to complete the proof of Theorem 2.1. We recall that $\Delta u=f(u)$ in $D$, and Lemma 2.4(a) and 2.7(b) together imply that

$$
\frac{1}{a^{3}} f\left(u\left(\frac{y_{1}+y_{2}}{2}\right)\right) \geqslant\left(\frac{1}{2 b^{3}}+\frac{1}{2 c^{3}}\right) f\left(u\left(y_{1}\right)\right) \text {. }
$$

If we assume for the moment that $f$ is strictly monotone, then inequality (2.15) together with (2.2) yields

$$
\frac{1}{a^{3}} f\left(u\left(\frac{y_{1}+y_{2}}{2}\right)\right)>\left(\frac{1}{2 b^{3}}+\frac{1}{2 c^{3}}\right) f\left(u\left(\frac{y_{1}+y_{2}}{2}\right)\right) .
$$

Notice that because of Lemma 2.7(a) and the positivity of $f$ we have

$$
\frac{1}{a^{3}}>\frac{1}{2} \cdot \frac{1}{b^{3}}+\frac{1}{2} \cdot \frac{1}{c^{3}}
$$

and this contradicts the convexity of $1 / x^{3}$. If the function $f$ is not strictly monotone on $(0,1)$, we may approximate it by a strictly monotone one $f_{\varepsilon}(u):=f(u)+\varepsilon u^{+}$, where $\varepsilon>0$ is small. Then problem (1.1), (1.2) has an increasing sequence of quasiconcave approximating solutions $u_{\varepsilon}$. It is easy to see that $u_{\varepsilon} \leqslant u$ and that $\left|f_{\varepsilon}\left(u_{\varepsilon}\right)-f(u)\right| \leqslant \varepsilon$ in $B \backslash \bar{\Omega}$, where $B$ is a large ball containing $D$. Standard a priori estimates as in Nečas (1967) or Ladyzhenskaya and Ural'tseva (1968) show that $u_{\varepsilon}-u \rightarrow 0$ in $W^{2, p}(B \backslash \bar{\Omega})$ for all $p$ sufficiently large. This implies the uniform convergence of $u_{\varepsilon}$ to $u$ on $\mathbf{R}^{n}$. Therefore, in (2.18) 
below, we may pass to the limit as $\varepsilon \rightarrow 0$ and thereby complete the proof of Theorem 2.1.

$$
Q_{\varepsilon}\left(x_{1}, x_{2}\right)=u_{\varepsilon}\left(\frac{x_{1}+x_{2}}{2}\right)-\min \left\{u_{\varepsilon}\left(x_{1}\right), u_{\varepsilon}\left(x_{2}\right)\right\} \geqslant 0 \quad \text { on } \mathbb{R}^{n} \times \mathbb{R}^{n} .
$$

REMARK 2.1. The inequality in Lemma 2.7(b) will also lead to a contradiction for solutions of

$$
\Delta u=b_{1}(u)|\nabla u|^{2}+b_{2}(u),
$$

where $b_{1}$ and $b_{2}$ are monotone nondecreasing, and where $b_{2}$ is positive for positive $u$. In order to prove Lemma 2.3 for such an equation, one needs an additional assumption such as $\partial b_{1} / \partial u \geqslant 2 b_{1}$. Another generalization of this approach to degenerate quasilinear elliptic equations is described in Kawohl (1983c). Finally, notice that porous medium-type equations $-\Delta\left(u^{m}\right)+g(u)=0$ can be treated with our method by making the substitution $v=u^{m}$.

REMARK 2.2. The following results were previously known, and their proofs use related variants of our method. If $u \equiv 1$ in $\Omega_{1}$ and $u=0$ on $\partial \Omega_{0}$, where $\Omega_{1} \subset \subset \Omega_{0}$, and where both $\Omega_{1}$ and $\Omega_{0}$ are convex, and if $u$ satisfies

or

$$
\Delta u=0 \text { in } \Omega_{0} \backslash \Omega_{1},
$$

$$
\operatorname{div}\left(|\nabla u|^{p-2} \nabla u\right)=0 \quad \text { in } \Omega_{0} \backslash \Omega_{1},
$$

or

$$
\Delta u=f(u) \text { in } \Omega_{0} \backslash \Omega_{1} \text { with } f \text { positive and monotone, }
$$

then all the level sets of $u$ are convex sets. These results were derived by Gabriel (1957), Lewis (1977) and Caffarelli and Spruck (1982).

\section{Symmetry of the free boundary}

We intend to establish symmetry properties of the free boundary for symmetric domains $\Omega$. We call a domain $\Omega \subset \mathbb{R}^{n}$ convex in (direction) $x_{n}$ if, for any fixed point $x^{\prime}=\left(x_{1}, \ldots, x_{n-1}\right) \in \mathbf{R}^{n-1}$, the orthogonal line to the $(n-1)$-dimensional hyperplane $\left\{x_{n}=0\right\}$ through $\left(x^{\prime}, 0\right)$ intersects $\partial \Omega$ at most twice.

THEOREM 3.1. If $f$ satisfies the assumptions of Proposition 1.1 or 1.2 , and if $\Omega \subset \mathbf{R}^{n}$ satisfies

$\mathbf{\Omega}$ is symmetric with respect to the $(n-1)$-dimensional hyperplane $\left\{x=\left(x_{1}, \ldots, x_{n}\right) \in \mathbf{R}^{n} \mid x_{n}=0\right\}$ and convex in $x_{n}$, 
and if $u$ is a variational solution of the exterior problem (1.1), (1.2), then all the level sets $\Omega_{c}:=\left\{x \in \mathbf{R}^{n} \mid u(x)>c\right\}$ of $u$ satisfy (3.1), and, in particular, the support of $u$ is symmetric with respect to $\left\{x_{n}=0\right\}$. Furthermore, if $\Omega$ is a ball with center at the origin, then so also are all the level sets of $u$.

Proof. The proof is an application of Steiner symmetrization in $\mathbf{R}^{n}$ as described in Polya and Szegö (1952) and in Kawohl (1984a). If $u$ is a variational solution of $(1.1),(1.2)$, then $J(u)=J\left(u^{*}\right)$, where $u^{*}$ denotes the Steiner-symmetrization of $u$ with respect to $x_{n}$. If the variational solution is unique, as it is under the assumptions of Proposition 1.1, then $u$ has to equal $u^{*}$, and $u$ is symmetrically decreasing in $x_{n}$. Under the assumptions of Proposition 1.2, however, the variational solutions need not be unique. Nevertheless, it was shown by Friedman and Phillips (1984) that they are nested:

If $u$ and $v$ are two different variational solutions of (1.1), (1.2), then either $u>v$ in $\{v>0\}$ and $\operatorname{supp} u \supset \operatorname{supp} v$, or $v>u$ in $\{u>0\}$ and $\operatorname{supp} v \supset \operatorname{supp} u$.

In the context of symmetrization, property (3.2) is a good substitute for uniqueness. This was observed in Kawohl (1983b). Let us now suppose that $u \neq u^{*}$ in contrast to Theorem 3.1. Then $u$ and $u^{*}$ form two different variational solutions of (1.1), (1.2), and either $\operatorname{supp} u \supset \operatorname{supp} u^{*}$ or $\operatorname{supp} u^{*} \supset \operatorname{supp} u$. Either case implies that $\operatorname{supp} u=\operatorname{supp} u^{*}$, since $u$ and $u^{*}$ are equimeasurable. Hence $u=u^{*}$, which proves Theorem 3.1.

REMARK 3.1. Notice that the result of this paragraph does not follow from the work of Gidas, Ni and Nirenberg (1979).

\section{The interior problem}

The corresponding interior problem

$$
\begin{gathered}
\Delta u=\lambda f(u) \quad \text { in } \Omega \subset \mathbb{R}^{n}, \lambda>0, \\
u=1 \text { on } \partial \Omega,
\end{gathered}
$$

was recently studied by Friedman and Phillips. In this case one is interested in the so called "dead core" $\{x \in \Omega \mid u(x)=0\}$. By a method which is totally unrelated to ours, they proved that for $n=2$ and convex $\Omega$ the dead core has to be convex. This result has been extended to arbitrary dimensions in Kawohl (1984b). Estimates of the size of the dead core may be found in Bandle, Sperb, Stakgold (1984). The symmetry result Theorem 3.1 has an obvious analogue for solutions 
of the interior problem. To prove this analogous result, one has to show that $(1-u)=(1-u)^{*}$. A weaker symmetry result for solutions of the interior problem was stated in Friedman and Phillips (1984), Theorem 1.10, where it was shown that the minimal and maximal solutions of problem (4.1), (4.2) on a ball do not depend on the angular coordinates. Notice that our result implies that $\partial u / \partial r \geqslant 0$ for variational solutions on balls.

\section{Acknowledgement}

This research was done at the Lefschetz Center for Dynamical Systems, Brown University, in Providence, RI, and financially supported by the Deutsche Forschungsgemeinschaft (DFG). I am gratefully indebted to both institutions. I also wish to thank C. Bandle and J. I. Diaz for bringing their work to my attention.

\section{References}

C. Bandle, R. Sperb and I. Stakgold (1984), 'Diffusion reaction with monotone kinetics', Nonlinear Anal. 8, 321-333.

J. I. Diaz and M. A. Herrero (1981), 'Estimates on the support of the solution to some nonlinear elliptic and parabolic problems', Proc. Roy. Soc. Edinburgh 89A, 249-258.

L. A. Caffarelli and J. Spruck (1982), 'Convexity properties of solutions to some classical variational problems', Comm. Partial Differential Equations 7, 1337-1379.

A. Friedman and D. Phillips (1984), 'The free boundary of a semilinear elliptic equation', Trans. Amer. Math. Soc. 282, 153-182.

L. S. Frank and W. D. Wendt (1984), 'On an elliptic operator with discontinuous nonlinearity', $J$. Differential Equations 54, 1-18.

R. Gabriel (1957), 'A result concerning convex level surfaces of 3-dimensional harmonic functions', $J$. London Math. Soc. 32, 286-294.

B. Gidas, W. M. Ni and L. Nirenberg (1979), 'Symmetry and related properties via the maximum principle', Comm. Math. Phys. 68, 209-243.

B. Kawohl (1983a), 'Starshapedness of level sets for the obstacle problem and for the capacitary potential problem', Proc. Amer. Math. Soc. 89, 637-640.

B. Kawohl (1983b), 'Starshaped rearrangement and applications' (LCDS Report 83-20, to appear in Trans. Amer. Math. Soc.).

B. Kawohl (1983c), 'Geometrical properties of level sets of solutions to elliptic problems' (LCDS Report 83-21, to appear in Nonlinear Functional Analysis and Its Applications, Proceedings of Symposia in Pure Math., Vol. 45, part 2, 25-36, Amer. Math. Soc., Providence, R. I., 1986.)

B. Kawohl (1984a), 'On the isoperimetric nature of a rearrangement inequality and its consequences for some variational problems' (LCDS Report 84-4, to appear in Arch. Ration. Mech. Anal.). 
B. Kawohl (1984b), 'More on convex level sets' (Report 114, Erlangen, appeared in revised form in Comm. Partial Differential Equations 10 (1985), 1213-1225).

O. Ladyzhenskaya and N. Ural'tseva (1968), Linear and quasilinear elliptic equations equations (New York, Academic Press).

J. L. Lewis (1977), 'Capacitary functions in convex rings', Arch. Rational Mech. Analysis 66, 201-224.

J. Nečas (1968), Les méthodes directes en théorie des équations elliptiques (Masson, Paris).

G. Polya and G. Szegö (1952), Isoperimetric inequalities in mathematical physics (Ann. Math. Studies, Vol. 27, Princeton University Press).

SFB 123

Universität Heidelberg

Im Neuenheimer Feld 294,

D6900 Heidelberg

West Germany 